\title{
reCHERches
}

Culture et histoire dans l'espace roman

4 | 2010

Les représentations du corps dans la litterature latinoaméricaine

\section{Le corps à corps avec le Pantanal}

Une lecture écocritique de l'œuvre poétique de Manoel de Barros

\section{Ludovic Heyraud}

\section{(2) OpenEdition}

\section{Journals}

Édition électronique

URL : https://journals.openedition.org/cher/8227

DOI : $10.4000 /$ cher.8227

ISSN : 2803-5992

Éditeur

Presses universitaires de Strasbourg

Édition imprimée

Date de publication : 30 juin 2010

Pagination : 273-284

ISBN : 978-2-35410-007-0

ISSN : 1968-035X

\section{Référence électronique}

Ludovic Heyraud, «Le corps à corps avec le Pantanal », reCHERches [En ligne], 4 | 2010, mis en ligne le 15 décembre 2021, consulté le 25 janvier 2022. URL : http://journals.openedition.org/cher/8227 ; DOI https://doi.org/10.4000/cher.8227

\section{cc) (i) (ㅇ)}

Ce(tte) œuvre est mise à disposition selon les termes de la Licence Creative Commons Attribution -

Pas d'Utilisation Commerciale - Partage dans les Mêmes Conditions 4.0 International. 


\title{
Le corps à corps avec le Pantanal: une lecture écocritique de l'œuvre poétique de Manoel de Barros
}

\author{
Ludovic Hevraud \\ Université Paul Valéry, Montpellier
}

\begin{abstract}
Cette communication va porter sur la vision du corps dans l'œuvre du Brésilien Manoel de Barros, le poète vivant le plus lu au Brésil. Né en 1916 à Cuiabá, dans l'état du Mato Grosso do Sul, il continue aujourd'hui encore, à l'âge de 92 ans, à écrire et publier des recueils d'une poésie alternativement versifiée ou en prose. Son œuvre se caractérise avant tout par un ancrage profond dans le Pantanal, la région dont il est originaire, à la frontière avec la Bolivie. Plus précisément, toute l'attention du poète se porte sur la relation existant entre l'Homme et le Pantanal, relation que nous avons qualifiée dans le titre de cette communication de véritable corps à corps. Nous verrons d'ailleurs que ce corps à corps induit une réinvention de la langue portugaise, de par la profusion de néologismes et d'entorses à la grammaire.
\end{abstract}

Notre réflexion se basera sur quatre de ses recueils, publiés entre 1985 et 1993: O Livro de Pré-Coisas (Le Livre des Pré-Choses), O Guardador de Águas (le Gardeur d'Eaux), Concerto a Céu Aberto para Solos de Ave (Concert à Ciel Ouvert pour Solos d'Oiseaux) et O Livro das Ignorãças, titre intraduisible puisque le mot ignorãças est un néologisme né de la fusion des substantifs «ignorância», l'ignorance, et «rã », la grenouille.

Notre étude se veut écocritique, l'écocritique étant, selon Cheryll Glotfelty (1996), «l'étude du rapport entre la littérature et l'environnement 
naturel». Selon cette approche, existant dans le domaine des littératures anglo-saxonnes depuis les années 60, mais encore embryonnaire dans le domaine des études lusophones, il convient d'analyser comment sont re-créés, re-présentés les éléments de la nature dans les œuvres littéraires, au moyen d'une véritable poétique écologique, qui puisse dire l'altérité de la nature, d'après Neil Evernden (1992), et prendre en compte le «travail écologique de l'écriture littéraire», selon Jonathan Bate (2000). Cette approche nous semble scientifiquement valide pour étudier le rapport au corps dans l'œuvre de Manoel de Barros, de par l'établissement dans sa poésie de véritables liens entre l'Homme et son environnement. Lígia Sávio (2004) explique d'ailleurs que «la poésie de Manoel de Barros a pour caractéristique une relation érotique avec la nature et avec la vie.» De plus, selon l'éditeur brésilien Carlos Augusto Viana, «se sédimente chez Manoel de Barros la conception du mot en tant qu'organisme vivant: le mot est végétal, le mot est animal.»

Notre objet sera donc d'observer comment, dans une région décrite comme un lieu originel, le poète met en scène les relations entre l'homme et son environnement. Celles-ci apparaissent d'emblée singulières. On peut citer le texte «lides de campear» («besogne des champs») du recueil Livro de Pré-Coisas:

Sente-se pois que árvores, bichos e pessoas têm natureza assumida igual. O homem no longe, alongado quase, e suas referências vegetais, animais. Todos se fundem na mesma natureza intacta. Sem as químicas do civilizado.

On sent donc que les arbres, les animaux et les gens ont une même nature assumée. L’homme au loin, presqu'étiré, et ses références végétales, animales. Tous se fondent dans la même nature intacte. Sans les chimies du civilisé.

Les rapports entre l'homme et la nature sont donc envisagés comme des relations d'égalité et de continuité, et non comme des relations hiérarchisées et dichotomiques.

Dans ce cadre, nous verrons en premier lieu qu'apparaissent des contacts, sensuels, érotisants et féconds entre l'homme et la nature, et entre les éléments eux-mêmes. Dans un second temps, que ces contacts vont jusqu'à l'osmose, la métamorphose. Nous verrons par ailleurs que ces contacts et métamorphoses s'opèrent chez Manoel de Barros également avec les mots. Enfin, nous nous demanderons ce qu'induit cet univers barrosien du point de vue du rapport au corps et au monde. 


\section{Contacts}

On observe dansl'ensemble del'œuvre de Manoel de Barrosl'établissement de contacts, tout à la fois entre les éléments naturels et entre les éléments naturels et l'homme. Ainsi, comme on peut le voir dans le recueil Concerto a céu aberto para solos de ave (que nous nommerons désormais Concerto), se nouent entre des éléments naturels des relations de séduction: Le texte appelé "Caderno de andarilho»(Cahier de vagabond) est un recueil de fragments prétendument écrits par le grand-père du sujet poétique, alors qu'il avait décidé de vivre dans les arbres, fragments que le je poétique aurait retrouvés plus tard, dans un vieux cahier. Dans ces fragments, écrits par un individu se fondant dans son environnement, on peut lire «Nódoas de muro seduzem caracóis» («Les tâches des murs séduisent les escargots»). Et la relation se fait plus intense dans ce vers extrait du huitième texte de la série intitulée «Seis ou treze coisas que eu aprendi sozinho» («six ou treize choses que j'ai apprises tout seul») du recueil O Guardadorde Águas: "as cigarras se perdem de amor pelas árvores» («Les cigales sont éperdues d'amour pour les arbres»). Et le rapport entre animal et minéral, et plus précisément entre limace et pierre, est explicitement sexuel dans le dixième texte de cette même série:

$\mathrm{Na}$ áspera secura de uma pedra a lesma esfrega-se

$\mathrm{Na}$ avidez de deserto que é a vida de uma pedra a lesma escorre...

Ela fode a pedra.

Ela precisa desse deserto para viver.

Sur l'âpre sécheresse d'une pierre la limace se frotte

Sur l'avidité de désert qu'est la vie d'une pierre la limace

glisse...

Elle baise la pierre.

Elle a besoin de ce désert pour vivre.

Le poète corporise, dote de désirs et sexualise ainsi l'ensemble de la nature, indistinctement. On peut même constater que cette sexualisation donne parfois lieu à fécondation, fécondation d'autant plus singulière qu'elle survient entre espèces différentes, voire entre entités vivantes et non-vivantes. Ainsi, dans le texte «Narrador apresenta sua terra natal» («Le narrateur présente sa terre natale»), du Livro de Pré-Coisas, on peut lire:

Aqui é o Portão de Entrada para o Pantanal. [...]

Há um rumor de útero nos brejos que muito me repercute. 
Ici c'est la porte d'entrée du Pantanal. [...]

Il y a une rumeur d'utérus dans les marais qui m’impressionne beaucoup.

Et dans le texte «Agroval» du même recueil, le poète décrit une raie qui s'enterre dans le sol, pendant la saison sèche, et les processus naturels qui s'opèrent alors:

[...] Ali, por debaixo da arraia, se instaura uma química de brejo. Um útero vegetal, insetal, natural.

[...] Là, sous la raie, s’instaure une chimie du marais. Un utérus végétal, insectal, naturel.

Naissent littéralement du ventre de cette Terre-Mère des êtres hybrides, indéterminés, (désignés par des mots eux-mêmes hybrides), ainsi que des relations et des contacts, encore une fois entre animaux, végétaux et minéraux:

[...]Penso nos embriões dos atos. Uma boca disforme de rapa-canoa que começa a querer se grudar nas coisas. Rudimentos rombudos de um olho de árvore. Os indícios de ínfimas sociedades. Os liames primordiais entre paredes e lesmas. Também os germes das primeiras idéias de uma convivência entre lagartos e pedras.

[...] E ao cabo de três meses de trocas e infusões - a chuva começa a descer.

[...] É a pura inauguração de um outro universo. Que vai corromper, irromper, irrigar e recompor a natureza.

Je pense aux embryons des actes. Une bouche difforme de poisson «rapacanoa» qui commence à vouloir se fixer sur les choses. Des rudiments émoussés d'un œil d'arbre. Les indices de sociétés infimes. Les liens primordiaux entre murs et limaces. Et aussi les germes des premières idées d'une vie commune entre les lézards et les pierres.

[...] Et au bout de trois mois d'échanges et d'infusions - la pluie commence à descendre. [...] C'est la pure inauguration d'un autre univers. Qui va corrompre, envahir, irriguer et recomposer la nature.

Des contacts entre tous ces éléments corporisés naissent donc l’inédit, l'inconnu, l'informe, et de cet inconnu et de cet informe surgissent des relations elles-mêmes inédites d'interactions et de collaborations entre tous ces corps. L'impression ressentie à la lecture de notre première citation du Livro de Pré-Coisas se confirme, du point de vue cette «natureza assumida igual» entre tous les éléments du vivant.

Et ce constat est également valable si l'on observe les contacts existants entre le corps de l'homme et son environnement, dans l'œuvre barrosienne. Il convient en un mot de revenir sur les personnages que met en scène le 
poète dans ses recueils. Ceux-ci ont tous pour point commun de vivre dans une certaine marginalité, en harmonie totale avec la nature environnante, au point de s'y fondre de corps et d'esprit. On peut noter tout d'abord leur très forte relation à la terre, au sol. Il en est ainsi de Bernardo da Mata, «Bernardo de la Forêt», qui apparaît dans tous les recueils depuis le Livro de Pré-Coisas, dont la publication date de 1985. Dans le texte II du recueil O Guardador de Águas, Bernardo est présenté en étroite relation avec le sol et les animaux:

Esse é Bernardo. Bernardo da Mata. Apresento.[...]

Até os caranguejos querem ele para chão.[...]

É homem percorrido de existências[...]

Espraiado na tarde -

Como a foz de um rio - Bernardo se inventa...

Lugarejos cobertos de limo o imitam.

Celui-ci c'est Bernardo. Bernardo da Mata. Je présente. [...]

Même les crabes le veulent comme sol. [...]

C'est un homme parcouru d'existences. [...]

Répandu dans l'après-midi -

Comme l'embouchure d'un fleuve - Bernardo s'invente...

Les terrains recouverts d'alluvions l'imitent.

Des animaux, les crabes, le voudraient comme sol, c'est-à-dire qu'ils aimeraient pouvoir être en contact permanent avec lui, adhérer à lui. Dans le même temps, Bernardo est décrit comme vivant tout à la fois dans la nature et dans son imaginaire. Et ces «existences» qui parcourent ce personnage le font accéder à d'autres états, grâce à la libération de son corps, «répandu» comme l'embouchure d'un fleuve. On pourrait même dire, en utilisant la terminologie de Gilles Deleuze et Félix Guattari, que ces existences le parcourant provoquent une certaine déterritorialisation de son corps, dans le sens où l'on peut lire dans l'Anti-CEdipe (1972: 162) que «se déterritorialiser, c'est quitter une habitude, une sédentarité. Plus clairement, c'est échapper à une aliénation, à des processus de subjectivation précis». On peut d'ailleurs à notre avis rapprocher la vision du corps qui se dégage des poèmes de Manoel de Barros d'un autre concept deleuzo-guattarien, celui du "corps sans organe», c'est-à-dire un corps qui serait doté de capacités permanentes de modification, un corps ouvert - je cite l'ouvrage Mille Plateaux (1980: 198) - «à des connexions qui supposent tout un agencement».

D'un point de vue écocritique, ces phénomènes de contacts entre les éléments, ou entre l'homme et la nature, mettent en évidence de la part 
du poète une volonté de décentrement par rapport à une vision trop anthropocentrique du monde, décrite par Blanc, Chartier et Pughe (2008):

le concept de décentrement semble toucher à l'essence du travail écologique de la littérature, $[\ldots]$ dans la mesure où il met en avant la nécessité de réinventer continuellement les façons par lesquelles la nature humaine s'inscrit dans la nature non humaine.

Et pour décrire cette inscription nouvelle de l'humain dans le nonhumain, par le biais de la littérature, Hayles (1995) insiste sur les deux notions de positionnalité et d'interactivité, en expliquant que, si l'homme est au monde, il est en même temps sujet et objet, et son destin n'est donc pas étranger au destin du monde. Ainsi, le statut mouvant du corps de Bernardo le fait-il participer au monde et du monde. Il permet en outre de constater ces relations d'interactivité avec une nature elle-même dotée d'un corps, ainsi qu'une positionnalité d'égal à égal avec celle-ci, après que s'est opéré un décentrement. L'écocritique et la conception du corps de Deleuze et Guattari trouvent ici leur point de jonction, puisqu'on peut lire dans L'Anti-Edipe (1973: 28) à propos du concept de corps sans organe (CsO) précédemment évoqué que,

le corps sans organes amène à l'idée d'un sujet qui «s'étale sur le pourtour du cercle dont le moi a déserté le centre».

Manoel de Barros va plus avant dans les rapports entre le corps de l'homme et la nature, à l'image des relations érotiques et sexuelles mis en scène entre les éléments eux-mêmes. Ainsi, dans le texte I de la première partie du Livro das Ignorãças intitulée "uma didáctica da invenção» («une didactique de l'invention»), le personnage de Felisdônio donne-t-il des conseils pour

apalpar as intimidades do mundo.

palper les parties intimes du monde.

Et dans le texte VI de O Guardador de Águas, on apprend du personnage de Roupa-Grande ( Grand-habit»), que

Uma árvore espera filhos dele.

Un arbre attend des enfants de lui.

Il faut noter ici que le substantif «árvore», est féminin en portugais, ce qui confère à cette filiation à venir une symbolique très forte de lien avec la Terre Mère, matérialisée par cet arbre. De plus, avec l'exemple de ce lien qui s'établit entre l'humain et le végétal, on voit pousser à son paroxysme 
l'osmose entre tous les êtres et toutes les choses. Cette osmose aboutit chez Manoel de Barros à des phénomènes de métamorphoses.

\section{Les métamorphoses}

Celles-ci sont en effet omniprésentes dans l'œuvre du poète, qu'elles soient présentées comme matérialisées et réalisées (et elles relèvent alors du merveilleux) ou comme spirituelles et désirées. On pourra ainsi dire de Manoel de Barros ce que Sartre (1949: 249) disait de la poésie d'Aimé Césaire:

Césaire $[\ldots]$ végétalise, animalise la mer, le ciel et les pierres. Plus exactement, sa poésie est un accouplement perpétuel de femmes et d'hommes métamorphosés en animaux, en végétaux, en pierres, avec des pierres, des plantes et des bêtes métamorphosées en hommes.

Le caractère innombrable des métamorphoses dans l'ensemble de l'œuvre de Manoel de Barros rend illusoire toute tentative d'une éventuelle typologie. On citera donc quelques exemples parmi tant d'autres révélateurs de ces phénomènes. Ainsi dans le Livro de Pré-Coisas, le je poétique retrouve-t-il un «tratado das metamorfoses» («traité des métamorphoses»), écrit par «un être irrésolu entre la branchette et le lézard» («um ente irresolvido entre vergôntea e lagarto»). Outre l'allusion directe aux Métamorphoses d'Ovide, qui sont de toute évidence l'un des livres de chevet de Manoel de Barros, on peut lire par exemple, dans une suite de fragments qui le doivent quant à eux à Héraclite:

Essa abulia vegetal sapal pedral - não será de ele ter sido ontem árvore?

Cette hésitation végétale crapale pierrale, ne viendrait-elle pas du fait qu'hier il était un arbre?

L'irrésolution de cet individu dont on ne sait rien dévoile ainsi un univers en constante mutation, peuplé d'êtres instables, aux corps en transformation. Ce qu'on peut donc qualifier de "capacité» à se transformer, réellement ou symboliquement, sous-tend à notre avis l'idée d'une altération de la conscience de l'homme. On peut citer à ce propos José Gil (1997: 185):

Les devenirs - devenir-animal, devenir-femme, devenir-autre - ne montrent pas seulement comme il est nécessaire de penser le corps comme virtuel, mais aussi comme non-humain, végétal, minéral, étranger à soi-même au plus profond de soi-même. [...] Le corps est profondément virtuel, c'est-à-dire inconscient. Le corps est pouvoir de transformation et devenir - devenir sensitif, affectif - qui atteint et désorganise l'unité de la conscience. 
Or, différents personnages des recueils $O$ Guardador de Águas et $O$ Livro das Ignorãças connaissent des devenirs autres qu'humains. Bernardo da Mata (dans le texte VI de O Guardador de Águas) «um dia chegou em casa árvore» («un jour est arrivé chez lui arbre»), tandis que le je poétique du Livro das Ignorãças explique, dans le texte XIII: «Estou atravessando um período de árvore» («je traverse en ce moment une période d’arbre»). Par ailleurs, dans le texte IX de la première partie de ce même recueil, «uma didáctica da invenção», le personnage de Felisdônio détaille de façon explicite le cheminement pour entrer «en état d'arbre»:

Para entrar em estado de árvore é preciso partir de um torpor animal de lagarto às três horas da tarde, no mês de agosto.

Em dois anos a inércia e o mato vão crescer em nossa boca.

Sofreremos alguma decomposição lírica até o mato sair na voz.

Hoje eu desenho o cheiro das árvores.

Pour entrer en état d'arbre il faut partir d'une torpeur animale de lézard à trois heures de l'après-midi, au mois d'août.

En deux ans l'inertie et la forêt vont croître dans notre bouche.

Nous subirons une certaine décomposition lyrique jusqu'à ce que la forêt sorte dans la voix.

Aujourd'hui je dessine l'odeur des arbres.

On voit donc ici clairement exposée la métamorphose, pour le moins mentale au départ (Manoel de Barros évoque "un état d'abre»), puisque débutant par une altération de la conscience du sujet, qui se trouve en situation de «torpeur animale». Cette métamorphose correspond à une véritable initiation, constituée par un double mouvement opposé de décomposition de «l'ancien corps» et de croissance de la forêt dans la voix. Enfin, cette initiation débouche sur un savoir, ou plutôt sur l'acquisition d'une nouvelle capacité, exprimée par la synesthésie finale: «dessiner l'odeur des arbres».

On peut également citer le personnage de «Sombra-Boa» («BonneOmbre») dans le texte «mundo pequeno» du Livro das Ignorãças:

Quando o rio está começando um peixe, ...

Ele me coisa

Ele me rã 
Ele me árvore, $[\ldots]$

Quand le fleuve commence un poisson, Il me chose

Il me grenouille,

Il m'arbre, [...]

Le fleuve a donc une puissance fécondante, et la profusion, le renouvellement de la nature, provoquent des métamorphoses chez le personnage de Sombra-Boa, une fois encore en animal, en végétal, et tout simplement, génériquement, en «chose». L'apparition d'un nouveau procédé poétique, la transformation de substantifs en verbes, qui plus est conjugués, est une façon supplémentaire d'effectuer des métamorphoses au sein même de la langue. La prise de conscience de la prolifération de la vie dans la nature a ici deux conséquences directes: la chosification du corps du personnage et une métamorphose des mots telle que décrite par Sartre dans son analyse de la poésie de Césaire.

La chosification, tout d'abord, est une des grandes thématiques de Manoel de Barros. Il faut surtout se garder de la confondre avec la notion de réification, signe d'assujettissement et d'humiliation. En effet, cette transformation du corps de l'homme en chose, qu'évoque Manoel de Barros dans sa poésie, a pour effet immédiat une libération donnant naissance à l'expression poétique. Dans la partie du recueil O Guardador de Águas intitulée "Retrato quase apagado em que se pode ver perfeitamente nada", ("portrait presqu'effacé où l'on peut voir le rien parfaitement»), observons le texte VI:

No que o homem se torne coisal, corrompem-se nele os veios comuns do entendimento.

Um subtexto se aloja.

Instala-se uma agramaticalidade quase insana, que empoema o sentido das palavras.

Aflora uma linguagem de defloramentos, um inauguramento de falas

Coisa tão velha como andar a pé

Esses vareios do dizer.

Dans ce en quoi l'homme devient chosal, s'altèrent en lui les voies habituelles de l'entendement.

Un sous-texte se loge.

S'installe une agrammaticalité presque insensée, qui poémise le sens des mots.

Affleure un langage de déflorations, une inauguration de paroles une chose aussi vieille que marcher à pied ces égarements du dire 
Ce qu'on pourrait entendre comme un abaissement de l'homme au niveau des choses est ici, paradoxalement, une élévation: le fait de devenir une chose corrompt sa perception, puis son expression. Naît alors un processus subversif et une nouvelle fois fécond en paroles nouvelles, poétiques et délirantes. Et le je poétique d'affirmer que ce phénomène n'est en rien rare ou novateur, mais au contraire aussi ancien, pour ne pas dire aussi ancestral, que le fait on ne peut plus naturel de marcher à pied. Le message du poète est ainsi parfaitement explicite: la «chosification» de l'homme est une libération, et elle est aussi vieille que l'homme.

Ensuite, la métamorphose des mots, précédemment évoquée, apparaît directement expliquée dans le texte VIII de la même sous-partie du recueil O Guardador de Águas, texte que l'on peut qualifier de méta-poétique:

Nas Metamorfoses, em duzentas e quarenta fábulas,

Ovídio mostra seres humanos transformados em pedras, vegetais, bichos, coisas.

Um novo estágio seria que os entes já transformados

Falassem um dialeto coisal, larval, pedral etc.

Nasceria uma linguagem madruguenta, adâmica, edênica, inaugural -

Que os poetas aprenderiam - desde que voltassem às

crianças que foram

Às rãs que foram

Às pedras que foram.

Dans les Métamorphoses, en deux cent quarante fables,

Ovide montre des êtres humains transformés en pierres, végétaux, animaux, choses.

Une nouvelle étape serait que ces êtres déjà transformés

parlent un dialecte de chose, de larve, de pierre, etc.

Naîtrait un langage d'aube, adamique, édénique, inaugural -

Que les poètes apprendraient - dès qu'ils reviendraient aux

enfants qu'ils étaient

aux grenouilles qu'ils étaient

aux pierres qu'ils étaient.

Est prononcé le souhait que les êtres métamorphosés par le biais de la représentation poétique s'expriment dans une langue elle-même métamorphosée, une langue originelle qui serait celle des poètes opérant un effort, que l'on qualifiera davantage d'effort de retour que d'effort de régression, puisqu'il n'est en aucun cas jugé négativement par le poète. La surprise naît ici du fait que l'on pourrait s'attendre à plus de rationalisme dans un poème métalittéraire. Or, il présente une "généalogie inédite» des hommes, en évoquant les grenouilles et les pierres qu'ils ont été. En l'espèce, 
Manoel de Barros parle des poètes, mais dans le sens où ceux-ci auraient la capacité de revenir à ce qu'était auparavant l'Homme au sens le plus large.

Dans l'œuvre poétique de Manoel de Barros, les contacts permanents avec la nature et les métamorphoses de l'homme et de la nature, qu'elles soient présentées comme réelles ou comme symboliques, induisent une union et parfois une fusion entre les deux. Celles-ci ont pour conséquence de provoquer chez les individus décrits une modification, une relativisation de leur conscience, un certain oubli de soi, et font de l'homme un être en devenir, fait de potentialités. Nous pourrons, pour conclure, citer deux exemples de métamorphoses symboliques, dévoilant justement un besoin de s'oublier, de se perdre ou, plus exactement, de ne pas se trouver. Le premier exemple est autobiographique, il est extrait du texte XIV de la partie «Mundo pequeno» («Petit monde») du Livro das Ignorãças:

De 1940 a 1946 vivi em lugares decadentes onde o mato e a fome tomavam conta das casas, dos seus loucos, de suas crianças e de seus bêbados. Ali me anonimei de árvore. [...]

Eu precisava de ficar pregado nas coisas vegetalmente e achar o que não procurava. [...]

De 1940 à 1946 j'ai vécu dans des endroits décadents où la forêt et la faim prenaient soin des maisons, de leurs fous, de leurs enfants et de leurs ivrognes.

Là-bas je me suis anonymé en arbre. [...]

J'avais besoin de rester cloué dans les choses végétalement et de trouver ce que je ne cherchais pas.

Le second exemple est tiré du texte $\mathrm{V}$ du «caderno de apontamentos» («Cahier de notes») du grand-père du sujet poétique dans le recueil Concerto:

Passei anos me procurando por lugares nenhuns.

Até que não me achei - e fui salvo.

Às vezes caminhava como se fosse um bulbo.

J'ai passé des années à me chercher dans des nulles parts.

Jusqu'à ce que je ne me trouve pas - et je fus sauvé.

Parfois je cheminais comme si j'étais un bulbe.

Les métamorphoses symboliques en arbre ou en bulbe se trouvent associées tour à tour à la nécessité ressentie de trouver ce qu'on ne cherche pas, et ensuite de se chercher sans - heureusement - se trouver. L'univers créé par Manoel de Barros est donc un univers où existe, pour les corps, la 
liberté de se transformer, de se métamorphoser, d'entrer en interaction, en contact, en communication avec une nature elle-même corporisée, de s'y fondre, voire de la féconder. Tout cela afin de répondre, selon nous, aux besoins de l'individu de s'oublier, d'être un autre, de n'être plus personne, de disparaître, de se fondre avec son environnement, d'y vivre en harmonie, libéré de toute psychologie et de tout sentiment, pour simplement sentir et pleinement être. Cette vision de l'Homme coïncide avec le concept encore une fois deleuzo-guattarien d' "heccéité» - c'est-à-dire d'individuation sans sujet - défini dans Mille Plateaux (1980: 343-344) où l'on peut lire que, quand on atteint cet état d'heccéité,

alors on est comme de l'herbe: on a fait du monde, de tout le monde un devenir, parce qu'on a supprimé de soi tout ce qui nous empêchait de nous glisser entre les choses, de pousser au milieu des choses.

\section{Bibliographie}

Bate, Jonathan, 2000, The song of the earth, Cambridge, Harvard Univ. Press.

Blanc, Nathalie, Chartier, Denis et Pughe, Thomas, 2008, "Littérature et écologie: vers une écopoétique", Écologie et politique, n 36, p. 17-28.

Deleuze, Gilles et Guattari, Félix, 1973, L’Anti-CEdipe, Paris, Minuit.

Deleuze, Gilles et Guattari, Félix, 1980, Mille Plateaux, Paris, Minuit.

Evernden, Neil, 1992, The social creation of nature, Baltimore \& Londres, The John Hopkins Univ. Press.

Gil, José, 1997, Metamorfoses do corpo, Lisboa, Relógio d’Água.

Glotfelty, Cheryll, 1996, The ecocriticism reader, Athènes \& Londres, Univ. of Georgia Press.

Hayles, N. K., 1995, «Searching for common ground», in M.E. Soulé et G. Lease, Reinventing nature? Responses to postmodern deconstruction, Washington D.C., Island Press, p. 47-63.

Sartre, Jean-Paul, 1949, «Orphée noir», Situations, III, Paris, Gallimard.

Sávio, Lígia, 2004, "A Poética de Manoel de Barros: uma sabedoria da terra», Literatura y lingüística, $\mathrm{n}^{\circ} 15, \mathrm{p} .67-80$. 